\title{
Synergistic Effects of a Root-Endophytic Trichoderma Fungus and Bacillus on Early Root Colonization and Defense Activation Against Verticillium longisporum in Rapeseed
}

\author{
Fatema Binte Hafiz, ${ }^{1 \dagger}$ Narges Moradtalab, ${ }^{2}$ Simon Goertz, ${ }^{3}$ Steffen Rietz, ${ }^{3}$ Kristin Dietel, ${ }^{4}$ \\ Wilfried Rozhon, ${ }^{1}$ Klaus Humbeck, ${ }^{5}$ Joerg Geistlinger, ${ }^{1}$ Günter Neumann, ${ }^{2}$ and Ingo Schellenberg ${ }^{1}$ \\ ${ }^{1}$ Department of Agriculture, Ecotrophology, and Landscape Development, Anhalt University of Applied Sciences, 06406 Bernburg, \\ Saxony-Anhalt, Germany \\ ${ }^{2}$ Institute of Crop Sciences, University of Hohenheim, 70593 Stuttgart, Baden-Württemberg, Germany \\ ${ }^{3} \mathrm{NPZ}$ Innovation GmbH, Hohenlieth-Hof, 24363, Holtsee, Schleswig-Holstein, Germany \\ ${ }^{4}$ ABiTEP GmbH, Berlin, Germany \\ ${ }^{5}$ Institute of Biology, Plant Physiology Department, Martin-Luther-University Halle-Wittenberg, 06120 Halle (Saale), Saxony-Anhalt, \\ Germany
}

Accepted 8 February 2022.

\begin{abstract}
Rhizosphere-competent microbes often interact with plant roots and exhibit beneficial effects on plant performance. Numerous bacterial and fungal isolates are able to prime host plants for fast adaptive responses against pathogen attacks. Combined action of fungi and bacteria may lead to synergisms exceeding effects of single strains. Individual beneficial fungi and bacteria have been extensively studied in Arabidopsis thaliana, but little is known about their concerted actions in the Brassicaceae. Here, an in-vitro system with oilseed rape (Brassica napus) was established. Roots of two different cultivars were inoculated with well-characterized fungal (Trichoderma harzianum OMG16) and bacterial (Bacillus velezensis FZB42) isolates alone or in combination. Microscopic analysis confirmed that OMG16 hyphae entered root hairs through root hair tips and formed distinct intracellular structures. Quantitative PCR revealed that root colonization of OMG16 increased up to 10-fold in the presence of FZB42. Relative transcript levels of the ethylene- and jasmonic acid-responsive genes $P D F 1.2, E R F 2$, and $A O C 3$ were recorded in leaves by quantitative reverse transcription PCR to measure induced systemic resistance in tissues distant from the roots. Combined action of OMG16 and FZB42 induced transcript abundances more efficiently than single inoculation. Importantly, microbial priming reduced Verticillium longisporum root infection in rapeseed by approximately 100 -fold
\end{abstract}

${ }^{\dagger}$ Corresponding author: F. B. Hafiz; fatema.hafiz@hs-anhalt.de

Funding: This research work was supported by International Graduate Schools in Agricultural and Polymer Sciences (AGRIPOLY), European Commission-European Social Fund (EU-ESF), Funding-ID: ZS/2016/08/ 80644 and German Federal Ministry of Education and Research (BMBF) through the program BonaRes II (Project DiControl), FundingID: 031B0514B and D.

*The $\boldsymbol{e}$-Xtra logo stands for "electronic extra" and indicates there is supplementary material published online.

The author(s) declare no conflict of interest.

(c) (1) () $\odot$ Copyright $(2022$ The Author(s). This is an open access article

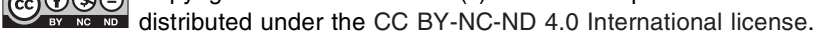

compared with nonprimed plants. Priming also led to faster and stronger systemic responses of the defense genes PDF1.2, ERF2, AOC3, and VSP2.

Keywords: fungal pathogen, gene expression, induced systemic resistance (ISR), intracellular interaction, root development, signal transduction, synergism, transcription, Trichoderma

Rhizosphere microbiomes play crucial roles in plant growth and health by improving nutrient mobilization and acquisition, exhibiting plant growth-promoting properties, enhancing plant tolerance to biotic and abiotic stresses, possessing antagonistic effects on phytopathogens and inducing plant defense-related genes (Berg et al. 2016; Mendes et al. 2011). The rhizosphere is inhabited by diverse plant growth-promoting microorganisms, including root-associated fungi (Coleman-Derr et al. 2016; Rodriguez et al. 2009). Many of those are known to stimulate the innate plant immune system against a wide range of pathogens (Alkooranee et al. 2015; Heil and Bostock 2002; Lee et al. 2012; Naznin et al. 2014). Trichoderma isolates (Ascomycota) are extensively used as plant-beneficial fungi because of their versatile abilities to stimulate ISR (induced systemic resistance) by modulating biosynthesis of numerous signaling molecules and hormones, including jasmonic acid (JA), ethylene (ET), and salicylic acid (SA) (Alkooranee et al. 2015; Brotman et al. 2013; Hermosa et al. 2012; Naznin et al. 2014). A number of Trichoderma strains have also been shown to enter root cells and develop an avirulent plant endo-symbiotic relationship (Geistlinger et al. 2015; Harman et al. 2004; Tucci et al. 2011). Due to the ability to form intracellular structures, some Trichoderma strains are categorized as root endophytes (Hoyos-Carvajal et al. 2009). Mutualistic characteristics of Trichoderma spp. have already been detected in a large number of plants including Arabidopsis thaliana (Alonso-Ramírez et al. 2015), cucumber (Meng et al. 2019), tomato (Coppola et al. 2019; Khoshmanzar et al. 2020), rapeseed (Poveda et al. 2019), and some woody plants (Carro-Huerga et al. 2020).

Besides plant growth-promoting fungi, plant rhizospheres also harbor communities of plant growth-promoting rhizobacteria 
(Kloepper et al. 1980; Windisch et al. 2021) with the ability to promote plant growth and suppress pathogens by means of direct antagonism or indirectly affecting pathogens via stimulating ISR (Chowdhury et al. 2015; Doornbos et al. 2012). Numerous rhizobacteria are able to enhance the root colonization ability of symbiotic fungi through synergistic interactions (FreyKlett et al. 2007). Bacillus velezensis FZB42, a well-studied gram-positive rhizobacterium (Fan et al. 2018), can trigger defense responses against Phytophthora nicotianae infection in Nicotiana benthamiana leaves by activating expression of the JA biosynthesis gene $L O X$, the ethylene-responsive transcription factor $E R F 1$, and the SA-regulated pathogen-related gene $P R-1 a$ (Wu et al. 2018). The root-endophytic nature of FZB42 was confirmed, using a green fluorescent protein-labeled strain (Fan et al. 2011, 2012). Sarosh et al. (2009) demonstrated enhanced expression of PDF1.2 upon Bacillus velezensis-mediated ISR in oilseed rape against Botrytis cinerea. The reported responses of plants primed with Bacillus velezensis include stimulation of resistance gene transcripts, disease suppression as well as increased root biomass. Nevertheless, potential synergistic effects of Trichoderma and Bacillus species in rapeseed and the underlying physiological mechanisms remain elusive.

In the last decades, oilseed rape has been extensively cultivated worldwide because of its growing importance as a feedstock for edible oil, a forage crop, and a source for renewable energy (Rybakova et al. 2017). The soil-borne pathogen Verticillium longisporum (C. Stark) can cause massive yield losses in rapeseed (Karapapa et al. 1997). Developing from microsclerotia, V. longisporum hyphae enter the host root system, preferentially via root hairs (Depotter et al. 2016), and subsequently colonize the root cortex and enter the xylem. Afterward, hyphae spread through the vascular system (Eynck et al. 2007) and eventually complete their life cycle by forming microsclerotia on stem and foliar tissues (Wilhelm 1955). The infestation by this soil-borne pathogen is vastly devastating in all Brassicaceae and is hard to eradicate by conventional fungicides (Depotter et al. 2016). Therefore, the aim of this study was to analyze the synergistic effects of Trichoderma harzianum OMG16 and Bacillus velezensis FZB42 in colonization of rapeseed roots, to monitor growth responses and changes in root morphology and to achieve induced systemic resistance against $V$. longisporum 43 (V143) infection. The presence of OMG16 fungus inside the root tissue was confirmed by microscopy as well as quantitative PCR (qPCR). The amount of root endophytic OMG16 and V143 from in vitro-grown plants were determined by qPCR. Finally, the potency of preventing infection of Brassica napus seedlings with $V$. longisporum by priming with combined application of OMG16 and FZB42 was studied. Induction of ISR in these plants was investigated by determination of the transcript abundances of selected rapeseed defense genes by applying quantitative reverse transcription PCR (qRT-PCR).

\section{RESULTS}

\section{Microscopic observation of root-fungus interactions.}

In order to confirm physical interactions between $T$. harzianum. and rapeseed roots, microscopic analysis was conducted $24 \mathrm{~h}$ after inoculation and on the next 2 days, which revealed that OMG16 spores germinated and hyphae were able to penetrate rapeseed root hairs. Chitin-specific staining revealed coillike structures inside the root hairs (Fig. 1B, C, and D), which confirmed that hyphae were formed inside the colonized plant cells. The micrographs shown in Figure 1 provide evidence that the OMG16 fungus used the root hair tips as an entry point in root cells. With respect to that, it is important to note that it was recently shown that fungi occasionally use root hair tips to enter plant cells (White et al. 2018). Taken together, these data confirmed the endophytic nature of OMG16 in rapeseed roots and its ability to develop distinct intracellular structures.

\section{Enhanced fungal root colonization rate in the presence of Bacillus velezensis FZB42.}

To assess synergistic interactions between $T$. harzianum and Bacillus velezensis, 2-week-old rapeseed roots from in vitro-cultivated plants were inoculated with either OMG16 or FZB42 alone or with both microorganisms in combination. Subsequently, the relative amount of the two microbes was quantified by qPCR at the same timepoints at which root colonization was observed by microscopic analysis. To investigate the impact of cocolonization on different cultivars, the Verticilliumsusceptible cultivar AgP1 and the tolerant cultivar AgP4 were included in this experiment. In comparison with single inoculation, the presence of FZB42 increased the amount of OMG16 that entered root cells of both cultivars (Fig. 2). The difference was highly pronounced on the first day, when the copy number of OMG16 was increased more than 10-fold in the cocultivation experiment, as compared with inoculation with the T. harzianum alone. However, also on the next 2 days, approximately three times more fungal DNA could be detected in coinoculated plants than in seedlings treated only with OMG16.

To assess whether acidification or alkalinization of the medium changed due to single and dual inoculation, OMG16, FZB42, and OMG16 plus FZB42 were grown separately in half-strength Murashige and Skoog (MS) medium and the $\mathrm{pH}$ was measured. We observed that FZB42 could acidify the growth medium by lowering the $\mathrm{pH}$ from 5.8 to 3.6 within 3 days. Also, in cultures coinoculated with OMG16 and FZB42, the $\mathrm{pH}$ declined rapidly, while in cultures inoculated with the fungus alone, decrease of the $\mathrm{pH}$ value was delayed (Supplementary Fig. S1). This might, at least partially, explain FZB42mediated growth enhancement of OMG16 since, as reported previously, Trichoderma growth is optimal in the acidic $\mathrm{pH}$ range (Pelagio-Flores et al. 2017).

In addition, the presence of OMG16 increased the root colonization rate of FZB42 significantly (Supplementary Fig. S2). In cultivar AgP1, the copy numbers of FZB42 were higher $(P<$ 0.05 on the first day and $P<0.0001$ on the second and third days) in coinoculated plants than in plants with single FZB42 inoculation. Moreover, in $\mathrm{AgP} 4$, the FZB42 copy number increased in coinoculated plants 2 days after inoculation (dai) $(P<0.01)$. This indicates that both microorganisms act synergistically to colonize the root system of B. napus.

\section{Effects on root morphology and growth.}

Several studies have reported that microorganisms can affect root growth and morphology. Thus, the effects of OMG16 and FZB42 were investigated in this study. Roots of 2-week-old rapeseed seedlings of equal sizes were treated with combined or single inoculation or were just treated with water as a control, and the subsequent changes in root morphology were digitized by optical scanning and were evaluated with the WinRHIZO software. The AgP4 cultivar had longer roots, prolonged fine roots, and a higher root mass and volume than the AgP1 cultivar (Supplementary Fig. S3). In the AgP4 cultivar, the development of root morphology was higher after single inoculation with OMG16 or FZB42 than in dual inoculation but only at the early timepoints. Coinoculation increased root lengths, dry weight, root volume, and fine-root lengths in early and late timepoints in the AgP1 cultivar. In this case, single inoculation had only minor effects on root morphology. (Supplementary Fig. S3). 


\section{Relative expression of rapeseed defense-related genes after OMG16 and FZB42 treatments.}

To analyze whether synergistic effects of OMG16 and FZB42 coinoculation might induce plant systemic resistance more efficiently than single microbe inoculation, 14-day-old rapeseed roots of both cultivars were treated with OMG16, FZB42, and OMG16 plus FZB42. The expression of JA/ET-responsive genes was measured in the leaves at four different timepoints, by
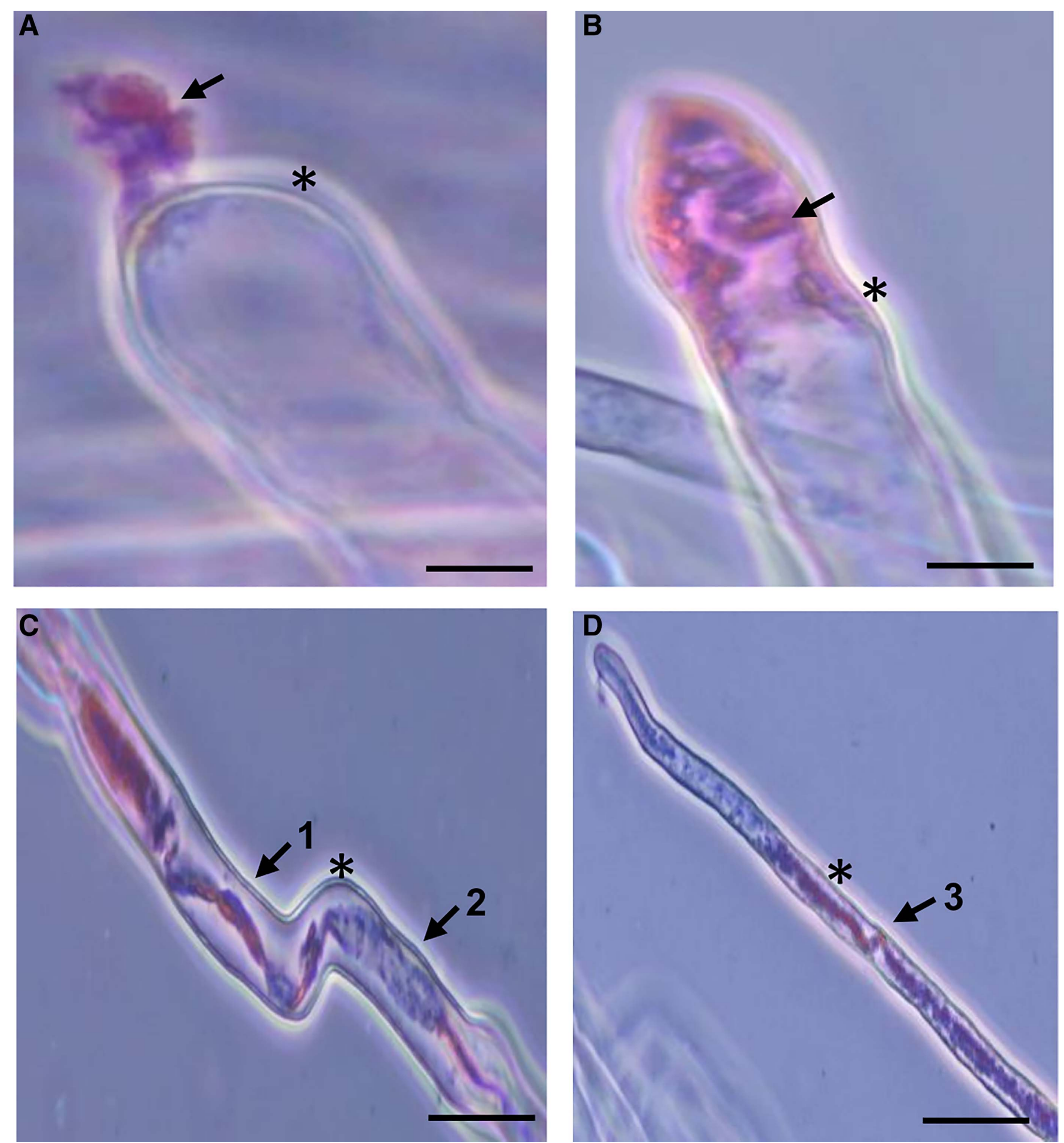

Fig. 1. Intracellular interactions between Trichoderma harzianum and rapeseed. A and B, OMG16 penetrating root hair tips. C and D, Root hairs endophytically colonized with OMG16. Arrows point to dense fungal cell wall (1), loosened fungal cell wall (2), coil-like structure (3) in hyphae stained with acid fuchsine; asterisks indicate cell walls of root hairs. Roots of 2-week-old plants were stained with acid fuchsine on days 1 (A, B), 2 (C), and 3 (D) after inoculation. Scale bars $=10 \mu \mathrm{m}$ (A to C) and $50 \mu \mathrm{m}$ (D). 
OMG16 enhanced PDF1.2 expression at 1 dai $(P<0.01)$ as compared with the untreated control plants (Fig. 3B). Relative expression of $P D F 1.2$ increased $(P<0.05)$, as well, in solely FZB42-treated plants at 3 dai. Transcript abundances were upregulated in OMG16 plus FZB42-treated plants at $2(P<0.01)$ and 3 dai $(P<0.05)$. Moreover, PDF1.2 expression was enhanced at 2 dai $(P<0.01)$ in OMG16 plus FZB42 coinoculated plants as compared with single FZB42 inoculation.

$E R F 2$ transcript levels increased in the AgP1 plants on day 2 after combined OMG16 and FZB42 treatment $(P<0.01)$ as compared with the untreated plants (Fig. 3C). On the other hand, in the AgP4 cultivar, relative transcript abundances of ERF2 increased in solely OMG16-treated plants at 1 hai $(P<$ $0.05)$ and $1(P<0.01)$ and 3 dai $(P<0.01)$. Inoculation with FZB42 alone enhanced the expression of ERF2 at 1 hai $(P<$ $0.05)$ and 1 dai $(P<0.01)$. ERF2 expression was upregulated at 1 hai $(P<0.001)$ and $1(P<0.0001)$ and 2 dai $(P<0.01)$ in coinoculated plants. Combined treatment of OMG16 and FZB42 enhanced ERF2 expression at 2 dai $(P<0.05)$ compared with single FZB42 application (Fig. 3D).

There were no significant differences in $A O C 3$ expression in the $\mathrm{AgP1}$ cultivar (Fig. 3E). In contrast, in $\mathrm{AgP} 4$ single fungal inoculation upregulated $A O C 3$ expression at 1,2 , and 3 dai $(P<$ 0.01 each) compared with the untreated plants. AOC 3 expression was also enhanced in single OMG16 treated plants at 2 dai $(P<$ 0.05), as compared with FZB42 treatment (Fig. 3F). Relative expression of $A O C 3$ increased in coinoculated plants at 1 hai $(P<0.05)$ and 2 and 3 dai $(P<0.01$ each $)$ compared with the untreated plants.

\section{Synergism of OMG16 and FZB42 reduced} $V$. longisporum V143 root colonization.

To this end, our results demonstrated that defense-related genes were induced in the B. napus $\mathrm{AgP} 4$ cultivar by inoculation with OMG16 and FZB42, while only a weak response was observed in the AgP1 variety. Thus, we decided to investigate the reaction in the $\mathrm{AgP} 4$ cultivar in more detail, particularly whether priming may reduce the root infection rates caused by $V$. longisporum Roots of 14-day-old B. napus cv. AgP4 seedlings were primed with OMG16 plus FZB42. Two days after priming, roots were infected with pathogenic $V$. longisporum V143. The absolute amount of V143 DNA present in total root DNA of primed plants was quantified by qPCR and was compared with the amount present in nonprimed plants. This indicated that the presence of V143 DNA was drastically reduced in primed plants, as compared with the nonprimed controls, at 1, 2, 3, and 5 dai ( $P<0.0001$ each) (Fig. 4A). Disease symptoms could not be observed due to the short growth period of the in-vitro plantlets.

To investigate the systemic defense responses of the plants, the relative transcript abundances of the previously used JA/ET marker genes $P D F 1.2, E R F 2$, and $A O C 3$ were analyzed in leaf tissue by qRT-PCR. In addition, expression of the JA-specific marker gene VSP2 (vegetative storage protein 2) was measured. Among the previously employed marker genes, PDF1.2 showed again the strongest response. PDF1.2 expression was higher at 1 dai $(P<0.01)$ in V143-infected primed plants than in the V143-infected nonprimed plants. In nonprimed V143infected plants, $P D F 1.2$ expression was enhanced at $1(P<$ $0.01), 2,3$, and 5 dai $(P<0.0001$ each) compared with the untreated control plants. $P D F 1.2$ expression was also enhanced in V143-infected primed plants at $1,2,3$, and 5 dai $(P<$ 0.0001 each) (Fig. 4B). Relative transcript abundances of ERF2 were significantly increased at $2(P<0.05)$ and 5 dai $(P<0.01)$ in primed plants compared with untreated controls. In contrast, in V143-infected, nonprimed plants ERF2 expression was enhanced only at 5 days $(P<0.01)$ after treatment (Fig. 4C). In primed plants, $A O C 3$ expression was already elevated at $2(P<0.01)$

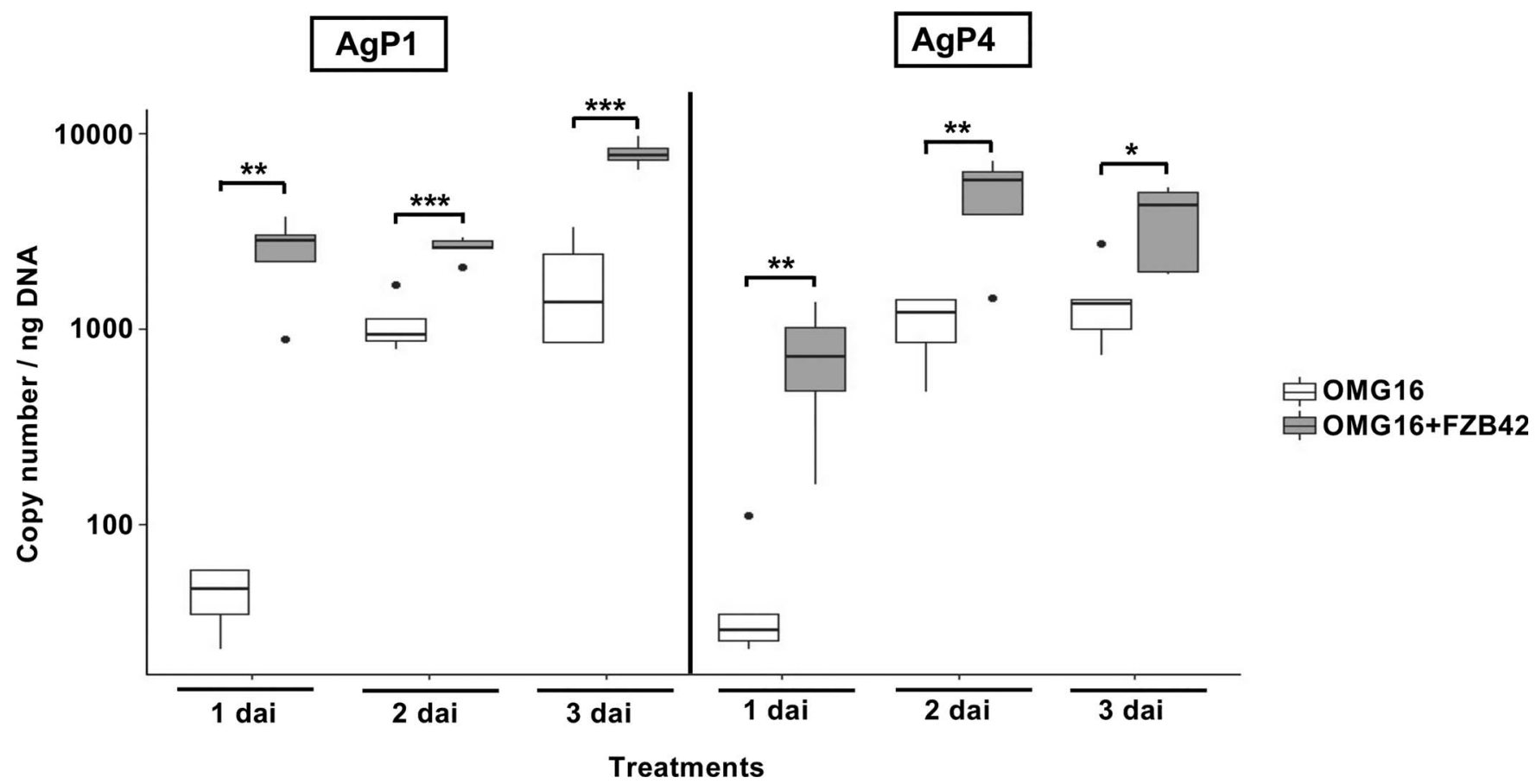

Fig. 2. Quantification of OMG16 in total root DNA. Two-week-old rapeseed roots of two cultivars (AgP1, left; AgP4, right) were inoculated with OMG16 and OMG16 plus FZB42. Copy numbers of OMG16 were determined by quantitative PCR (qPCR), using the absolute quantification method. Welch's $t$ test was employed at $P<0.05$ after performing the Shapiro-Wilk normality test $(n=5$, in quadruplicate qPCRs, except OMG16 at 2 and 3 days after inoculation (dai), OMG16+FZB42 at 3 dai in AgP1, and OMG16 at 2 dai in AgP4, where $n=4)$. All data sets were normally distributed except the copy number of OMG16 at 1 dai in AgP4. Therefore, Wilcoxon rank-sum test was employed, at $P<0.05$, in this sample. One asterisk (*) indicates $P<$ 0.05 , two $\left(^{* *}\right) P<0.01$, and three $\left(^{* *}\right) P<0.001$. OMG16 = Trichoderma harzianum OMG16, FZB42 = Bacillus velezensis FZB42. 
and 5 dai $(P<0.05)$ after V143 infection, while expression of AOC3 in nonprimed Vl43-infected plants started to increase only as late as 5 dai $(P<0.05)$ (Fig. 4D). To investigate activation of the JA pathway in more detail, we also included the VSP2 marker gene in this experiment, which showed enhanced expression at $1,2,3$, and 5 dai $(P<0.0001$ each $)$ in V143-infected primed plants, as compared with the untreated controls. VSP2 expression increased likewise at $1,2,3$, and 5 dai $(P<0.0001$ each) in nonprimed V143-infected plants. Moreover, higher expression of VSP2 was detected at 2 dai $(P<0.01)$ in V143infected primed plants than the V143-infected nonprimed plants (Fig. 4E).

\section{DISCUSSION}

Interaction between rapeseed roots and $T$. harzianum .

We have confirmed that $T$. harzianum OMG16 exhibits an intracellular endophytic lifestyle in rapeseed roots of
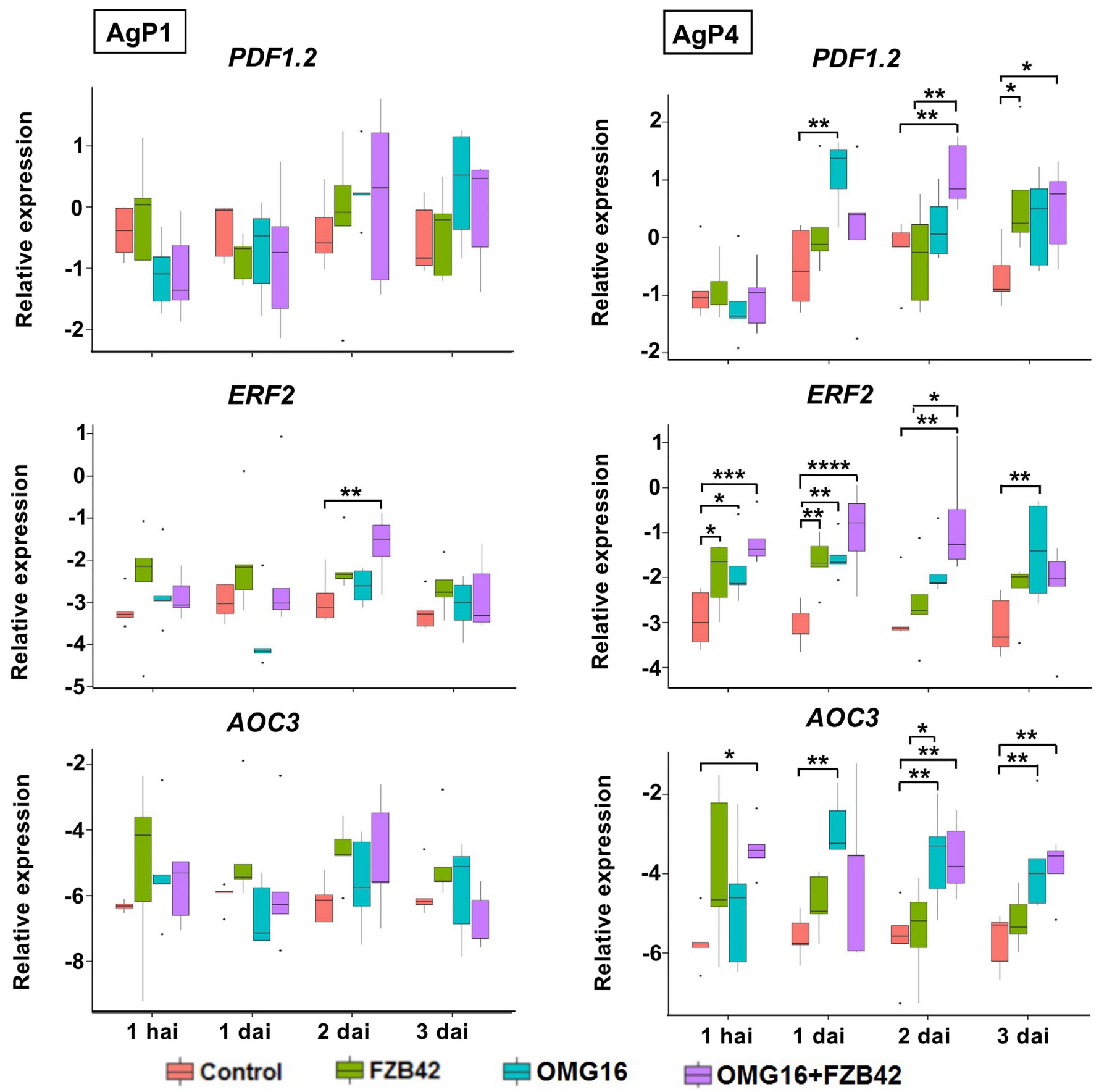

Fig. 3. Relative expression of rapeseed defense genes PDF1.2, ERF2, and AOC3 upon OMG16, FZB42, and OMG16 plus FZB42 inoculation. Twoweek-old rapeseed roots of two cultivars ( $\mathrm{AgP} 1$, left; $\mathrm{AgP} 4$, right) were inoculated, and relative transcript abundances were analyzed by quantitative reverse transcription PCR (qRT-PCR) in leaves at $1 \mathrm{~h}$ after inoculation (hai) and 3 consecutive days after inoculation (dai) of roots. BnaUbiquitin11, BnaActin, and BnaTubulin were used as endogenous controls for normalization. Box and whisker plots show quantification cycle values of five plants with quadruplicate qRT-PCRs, in which significance of gene expression was calculated by Dunnett's test, with multivariate testing adjustment at $P<$ 0.05 compared with the control plants. To compare the differences in gene expression upon OMG16, FZB42, and OMF16 plus FZB42 treatments, oneway analysis of variance with post hoc Tukey's highly significant difference tests were performed. One asterisk $(*)$ indicates $P<0.05$, two $(* *) P<$ 0.01, three $(* * *) P<0.001$, and four $(* * *) P<0.0001$. OMG16 = Trichoderma harzianum OMG16, FZB42 = Bacillus velezensis FZB42. 


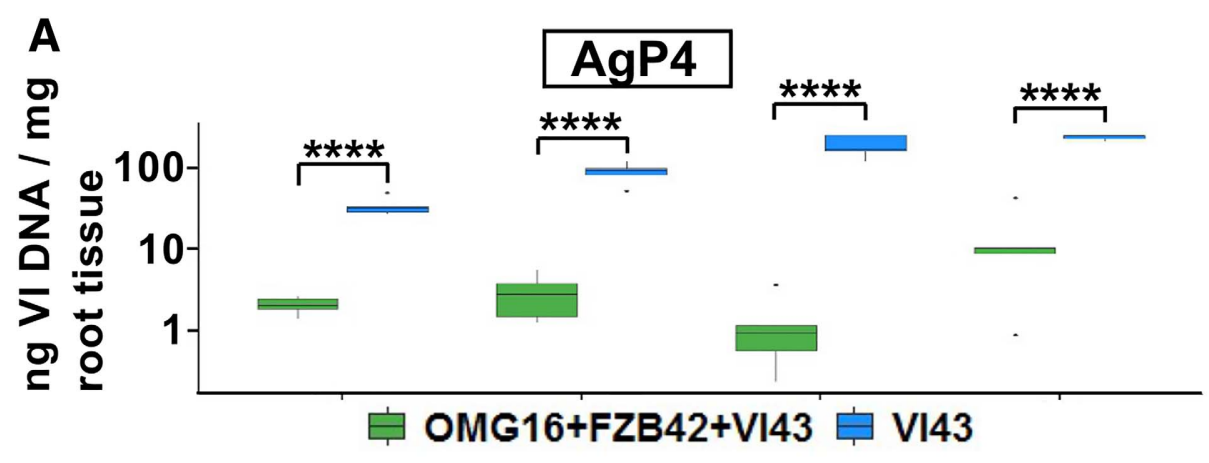

B
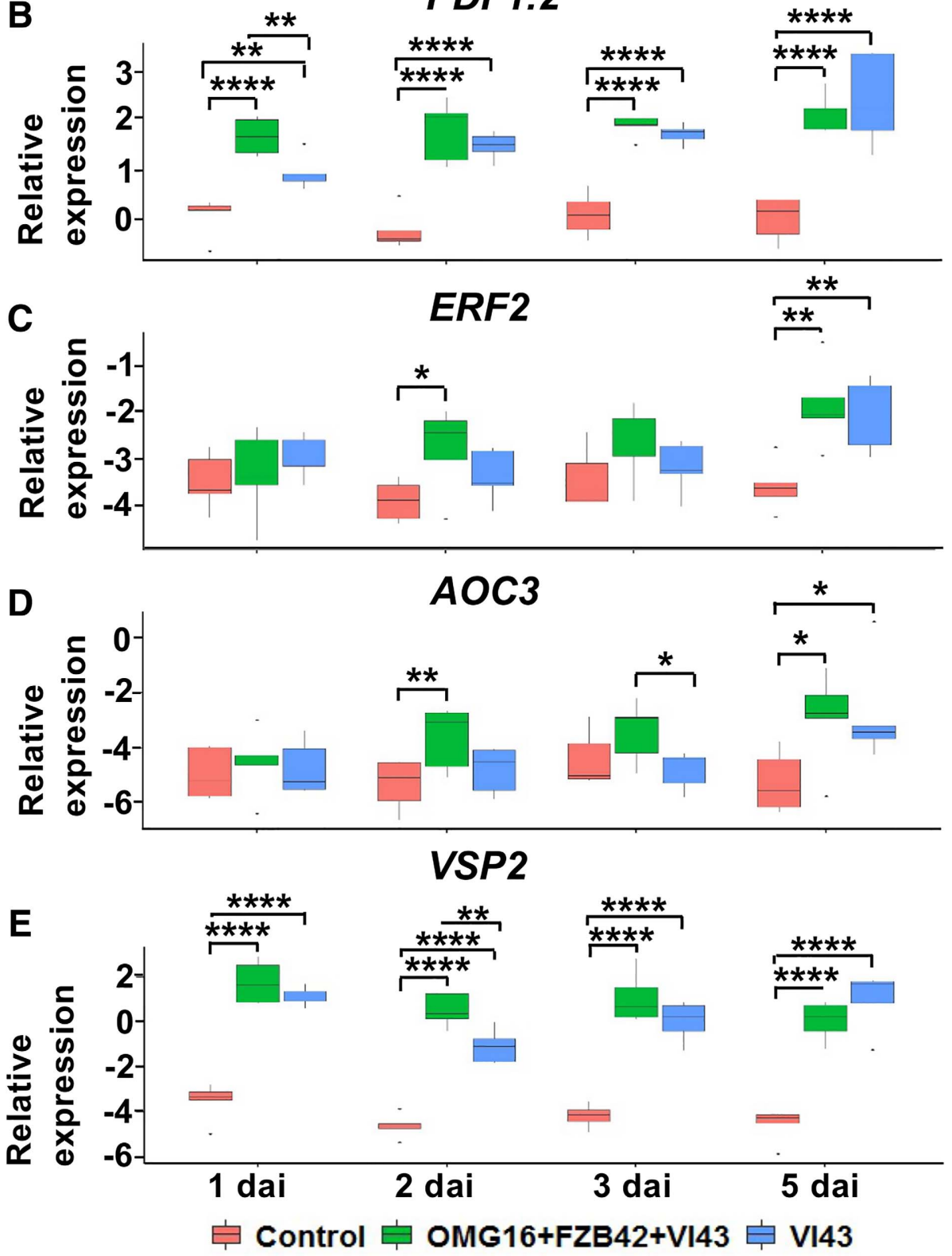

Fig. 4. Priming with OMG16 and FZB42 enhances the resistance of Brassica napus cv. AgP4 against infection with Verticillium longisporum. Roots of 2-weekold rapeseed plantlets of the AgP4 cultivar were primed with OMG16 plus FZB42. Two days after priming, roots were infected with V143. The absolute amount of V143 DNA in root samples was quantified by quantitative PCR, using a single locus genomic region of VI43. An unpaired $t$ test was performed at $P<0.05$ between OMG16 plus FZB42 plus V143 and V143 alone. A, Quantification of V143 DNA in total roots and B, relative expression of rapeseed defense genes PDF1.2, C, ERF2, D, $A O C 3$, and E, VSP2 upon OMG16 plus FZB42 plus V143 and V143 inoculation alone. To assess systemic responses, the relative transcript abundances of the rapeseed defense genes $P D F 1.2, E R F 2, A O C 3$, and VSP2 were analyzed by quantitative reverse transcription PCR (qRT-PCR) in leaves of the same plants tested in A. BnaUbiquitin11, BnaActin, and BnaTubulin were used as endogenous controls for normalization. Box and whisker plots show quantification cycle values of five biological replicates with quadruplicate qRT-PCRs where significance of gene expression was calculated by Dunnett's test with multivariate testing adjustment at $P<0.05$ compared with the control plants. Relative expression of genes between OMG16 plus FZB42 plus V143 and single V143 treatments was calculated by unpaired $t$ test at $P<0.05$. One asterisk $\left(^{*}\right)$ indicates $P<0.05$, two $\left(^{* *}\right) P<0.01$, three $\left(^{* * *}\right) P<0.001$, and four $\left({ }^{* * * *}\right) P<0.0001$. OMG16 $=$ Trichoderma harzianum OMG16, FZB42 = Bacillus velezensis FZB42, V143 = V. longisporum 43, dai $=$ days after infection. 
in vitro-grown plantlets, as visualized by microscopic analyses. Axenic OMG16 possessed the ability to penetrate root hairs shortly after inoculation, notably within $24 \mathrm{~h}$. In a previous study, microscopic observations detected $T$. harzianum CECT 2413 hyphae on tomato root surface at $24 \mathrm{~h}$ after inoculation (Samolski et al. 2012). In plant families other than the Brassicaceae, T. harzianum T-203 attached, to the root epidermis but not to root hair tips in cucumber, by forming appressoria-like structures mediated by TasHydl-encoded hydrophobin proteins (Yedidia et al. 1999; Viterbo and Chet 2006). T. harzianum CECT 2413 can also colonize the intercellular spaces of tomato roots without disrupting cell integrity and, subsequently, enters root epidermal cells and the cortex (Chacón et al. 2007; Velázquez-Robledo et al. 2011; Yedidia et al. 1999) while it undergoes morphological changes, such as formation of papilla-like swollen hyphal tips and yeast-like cells (Chacón et al. 2007). In our study, OMG16 bound high amounts of fuchsine red in the cell walls, which enabled us to distinguish densely compacted hyphae from hyphal cellwall structures of lower density (Fig. 1). These regions of lower cell-wall density may be considered as potential sites of molecular crosstalk (Harman et al. 2004; Yedidia et al. 2000) between the fungus and plant, which possibly led to transcriptional responses of the rapeseed defense-related genes expressed systemically in leaves. Appressoria-like structures on the root epidermis or cortex were not found, which provided further evidence that root hair tips were the main entering sites of OMG16 into the rapeseed root system.

\section{Effects of synergism on Trichoderma root colonization abundance.}

Quantification of OMG16 DNA in roots, using established protocols (Geistlinger et al. 2015; Poveda et al. 2019), demonstrated that the presence of FZB42 together with the fungus exhibited synergistic effects and significantly enhanced the Trichoderma root colonization rate (Fig. 2). Medium acidification caused by Bacillus velezensis FZB42 (Mpanga et al. 2020) might be a factor for improved root colonization frequency of $T$. harzianum OMG16 (Supplementary Fig. S1). In addition, other mechanisms might be relevant. For instance, Bacillus amyloliquefaciens enhances the expression of cellulase- and xylanase-encoding genes (Karuppiah et al. 2021) as well as plant defense-response suppressors (Karuppiah et al. 2019), which favorably prepare the cell walls of the roots for beneficial fungi to penetrate. In soilgrown maize, the root colonization rate of OMG16 successfully increased by $210 \%$ in the presence of a consortium of five Bacillus strains (Mpanga et al. 2019b). However, this effect depended largely on preferential ammonium supply together with the nitrification inhibitor DMPP (3,4-dimethylpyrazole-phosphate) (Mpanga et al. 2019a). This resembles the conditions in our study, in which Murashige and Skoog medium was used under sterile conditions, which prevents ammonium nitrification. Another important factor might be increased fine-root growth. Importantly, our data demonstrated that inoculation of B. napus with FZB42 stimulated fine-root growth (Supplementary Fig. S3C), which provided a larger root surface area for quicker and better OMG16 root colonization. With respect to that, it is important to mention that, particularly at early timepoints, significantly more fungal DNA could be detected in cotreated roots than in roots treated with OMG16 alone (Fig. 2), which substantiates the enlarged surface area hypothesis. Indeed, it has been previously shown that rhizosphere-competent bacteria can stimulate fine-root formation, which subsequently provides increased potential sites for plants and fungi to interact (Frey-Klett et al. 2007). Such effects have also been described for FZB42 (Thonar et al. 2017; Yusran et al. 2009).

\section{Effects of synergism on the expression}

\section{of defense-related rapeseed genes.}

We provide compelling evidence in Figure 2 for synergistic effects of Trichoderma and Bacillus species in colonization of B. napus roots. Analysis of gene expression by qRT-PCR (Fig. 3) at the same timepoints revealed a very slow and weak induction of the selected defense-related genes PDF1.2, ERF2, and $A O C 3$ in the AgP1 cultivar. In sharp contrast, expression of the analyzed genes was much faster and greater in the AgP4 cultivar, pointing also to genotypic differences in responsiveness of the selected rapeseed hybrids. In the experiment, the advantage of coinoculation became clearly visible in this cultivar at 2 dai. Expression of the ET marker gene ERF2 was increased at 1 hai and remained activated till 2 dai in dual-inoculated plants. Coinoculation also enhanced ERF2 expression more strongly than did the single bacteria. In a previous study, ET-responsive transcription factors (ERFs) belonging to a subfamily of the AP2/ ERF superfamily (Yang et al. 2016) were shown to directly regulate the activation of JA/ET-dependent defense genes and to play a vital role in A. thaliana resistance against necrotrophic pathogens.

$A O C 3$, which encodes a JA biosynthesis gene (Stenzel et al. 2012), was expressed at 1 hai and remained activated till 3 dai (except 1 dai) in coinoculated plants. The AOC3 enzyme catalyzes the formation of 12-oxo-phytodienoic acid, the first biologically active intermediate in jasmonate biosynthesis (Schaller et al. 2008). Tolerance against different biotic and abiotic stresses induced by the JA hormonal pathway through the activation of $A O C$ genes was achieved in A. thaliana (Stenzel et al. 2012), barley (Jacquard et al. 2009), wheat (Eltayeb Habora et al. 2013; Zhao et al. 2014), soybean (Wu et al. 2011), and Jatropha curcas (Liu et al. 2010).

The expression of $P D F 1.2$ also was significantly upregulated in OMG16 plus FZB42 coinoculated plants at 2 and 3 dai, as compared with the controls. Besides, PDF1.2 expression was higher at 2 days after dual inoculation than after single inoculation. Taken together, these data suggest that the JA and ET signaling pathways were activated in AgP4 plantlets shortly after combined microbial treatment ( 1 hai) and remained activated at least until 3 dai (Fig. 3B, D, and F), the end point of this experiment. A similar effect was reported in oilseed rape, in which Bacillus velezensis induced ISR by increasing JA/ET-dependent PDF1.2 expression against Botrytis cinerea (Sarosh et al. 2009). However, our gene-expression data suggest that combined application of root endophytic OMG16 and FZB42 can activate the rapeseed defense pathways more efficiently than inoculation with the fungus or bacterium alone.

\section{Priming effects on $V$. longisporum root infection in AgP4.}

To investigate whether priming may reduce the susceptibility of rapeseed roots for infection with V143, plants were primed with OMG16 plus FZB42 2 days prior to V143 infection. Subsequent quantification of VI43 DNA by qPCR demonstrated that primed plants were approximately 100 times less colonized by V143 than nonprimed plants. As shown by our microscopic studies (Fig. 1), OMG16 forms endophytic hyphae in B. napus root hairs, which may act as physical barriers for V143 hyphae to grow and penetrate the roots. A similar mechanism may also account for the FZB42 bacterial cells that had colonized the roots. Moreover, competition of OMG16 and FZB42 with V143 hyphae for minerals and nutrients might be an additional factor for V143 growth suppression. Previous studies showed that Trichoderma asperellum T34 protected tomato plants from fusarium wilt by iron competition (Segarra et al. 2010). Pichia guilliermondii suppressed Botrytis cinerea in apple (Zhang et al. 2011) and Colletotrichum spp. in chilli (Chanchaichaovivat et al. 2008) by competition for sugars and nitrate. Furthermore, 
hydrolytic enzymes produced by many Trichoderma strains may permeabilize and degrade the cell wall of fungal pathogens to facilitate the subsequent entry of secondary antimicrobial metabolites (Karlsson et al. 2017; Köhl et al. 2019). Recently it was shown that an effective biocontrol activity was achieved in vitro by a Trichoderma virens strain that produced bioactive siderophores (Angel et al. 2016) and membrane-attacking peptaibols (Palyzová et al. 2019). Similarly, Bacillus velezensis PGPBacCA1 was shown to secrete antifungal compounds including surfactin, iturin, fengycin, and bacillomycins into the interaction zones with the pathogens Macrophomina phaseolina (Torres et al. 2016), Fusarium oxysporum (Palyzová et al. 2019), and Verticillium dahliae (Han et al. 2015). Similar mechanisms as observed in this study might also produce an antifungal effect of combined OMG16 and FZB42 application to inhibit V143 growth.

Besides reducing V143 growth in root tissue, priming of rapeseed cultivar AgP4 with OMG16 and FZB42 also enhanced the plant systemic defense response against V143 infection (Fig. 4). Analysis of gene expression by qRT-PCR revealed a fast and strong induction of VSP2 and PDF1.2 in leaf tissues. Expression of these two defense marker genes indicated the activation of the JA/ET signaling pathways both in Vl43-infected primed and nonprimed plants. In addition, expression of the JA and ET marker genes $A O C 3$ and ERF2 reacted faster and stronger to
V143 infection in primed compared to nonprimed plants. In line with our results, a previous study showed that $T$. harzianum TH12 induces systemic resistance via the JA/ET pathways by increasing the ERF2, PDF1.2, and AOC3 gene expression levels at 1 and 2 dai in oilseed rape and thereby establishes systemic resistance against Sclerotina sclerotiorum (Alkooranee et al. 2017). Similar synergistic effects were observed in the combined application of T. harzianum Tr6 and Pseudomonas sp. strain Ps14, which significantly increased the resistance level in cucumber against $F$. oxysporum through elevated expression of a set of defense-related genes (Alizadeh et al. 2013).

\section{Conclusion.}

Our study has demonstrated that T. harzianum OMG16 and Bacillus velezensis FZB42 acted synergistically to induce the JA and ET hormone signal transduction pathways in rapeseed, which are crucial for activating ISR. Pretreatment of roots with both OMG16 and FZB42 restrict V143 infection in roots and systemically transduced fast, strong, and long-lasting signals based on the JA and ET hormonal pathways (Fig. 5). Due to the importance of early root development for field performance of oilseed rape (Koscielny and Gulden 2012; Louvieaux et al. 2020) and the continuously declining availability of chemical

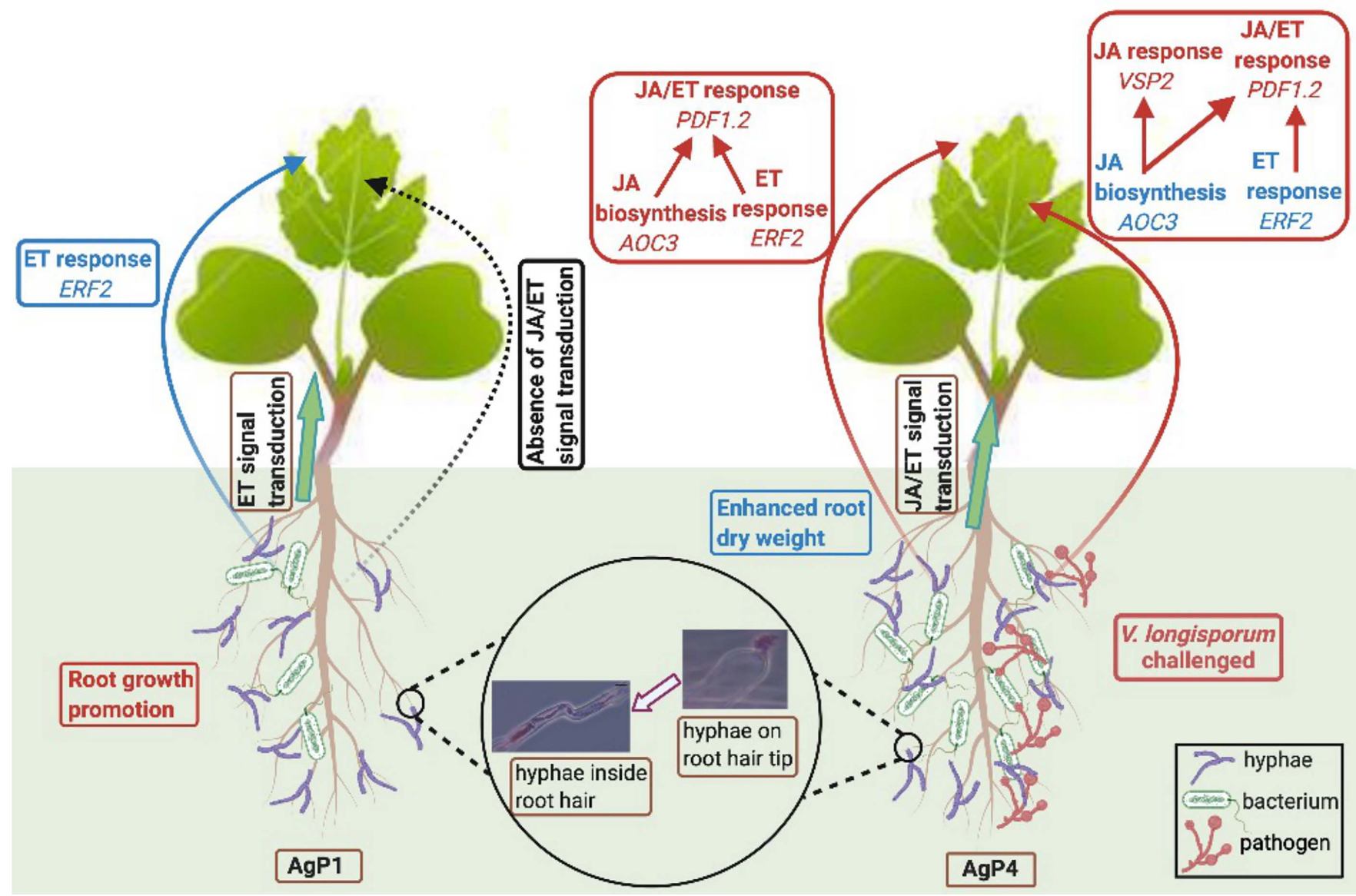

Fig. 5. Simplified model of molecular mechanisms involved in rapeseed defense signaling modulated by crosstalk between the ethylene (ET) and jasmonic acid (JA) hormonal pathways in response to Trichoderma-rapeseed interaction and Trichoderma-Bacillus-rapeseed synergism. Red represents faster and stronger while blue represents slower and weaker responses. In both cultivars, OMG16 hyphae attached to the top of root hair tips and, after penetration, developed distinct intracellular structures. Also root development was enhanced in coinoculated plants of both cultivars. In OMG16 and FZB42-coinoculated AgP1 plants, the ET signal was transduced from the root system to leaves, in which it induced expression of the ET-responsive gene ERF2. JA/ET signal transduction was absent after single OMG16 inoculation. In the AgP4 cultivar, combined treatment of OMG16 and FZB42 in roots transduced strong JA and ET signals, which induced expression of the JA/ET marker gene PDF1.2, the JA biosynthesis gene AOC3, and the ET-responsive ERF2 gene in leaves. In OMG16 and FZB42 primed plants, expression of the JA and ET marker genes was, in general, stronger and faster after V143 than in the controls. Figure created with BioRender. 
plant protection agents, our results may offer a perspective for the development of alternative and sustainable approaches to enhance the tolerance of rapeseed cultures against fungal infections. The results also suggest that cultivar-dependent differences in the responsiveness of host plants with respect to inoculant effects need to be considered in this context. This may, at least partially, explain the reported variability of plant responses to beneficial microbes frequently observed under field conditions.

\section{MATERIALS AND METHODS}

\section{Fungal and bacterial culture conditions.}

T. harzianum OMG16 strain (Moradtalab et al. 2020; Mpanga et al. 2019a, b) was obtained from the strain collection of Anhalt University of Applied Sciences, Bernburg, Germany. The fungus was grown on potato dextrose agar (PDA) (Carl Roth) at room temperature. Spores were harvested by adding $5 \mathrm{ml}$ of sterile deionized water to the surface of a 20-day-old PDA plate. The obtained spore suspension was filtered through a single layer of Miracloth (Merck), in order to separate mycelial fragments from conidia. The spore concentration was determined with the hemocytometer and was adjusted to a final concentration of 20,000 spores per milliliter. Fresh spore suspension of V143 with a concentration of $10^{6}$ spores per milliliter was provided by NPZ Innovation $\mathrm{GmbH}$, Hohenlieth, Germany. The fungus was grown in potato dextrose broth for 7 days, at $23^{\circ} \mathrm{C}$, on a rotary shaker at $100 \mathrm{rpm}$. The spore suspension was harvested by filtering through sterile gauze. Spore concentration was counted with a hemocytometer and was adjusted to $10^{6}$ spores per milliliter (Eynck et al. 2009).

The bacterial strain Bacillus velezensis FZB42 syn. Bacillus amyloliquefaciens subsp. plantarum FZB42 was provided by ABiTEP GmbH. A single colony of Bacillus velezensis FZB42 was picked to inoculate DEV nutrient medium (per liter: meat extract $10.0 \mathrm{~g}$, meat peptone $10.0 \mathrm{~g}$, and sodium chloride $5.0 \mathrm{~g}$, adjusted to $\mathrm{pH} 7.3$ with $\mathrm{NaOH}$ ). The culture was grown overnight at $37^{\circ} \mathrm{C}$ at $160 \mathrm{rpm}$ to an optical density at $600 \mathrm{~nm}$ $\left(\mathrm{OD}_{600}\right)$ of 0.2 in order to inoculate rapeseed roots.

\section{Plant materials, growth conditions, and microbial inoculation.}

Induction of defense-related genes caused by OMG16 and FZB42 separately or in combination was analyzed in two $B$. napus (oilseed rape) genotypes, $\mathrm{AgP} 1$ and $\mathrm{AgP} 4$. B. napus seeds were provided by the NPZ Innovation GmbH. According to the classification in Verticillium bioassays, AgP1 was more susceptible toward the pathogen in the juvenile stages than was AgP4 (Supplementary Fig. S4). Furthermore, the genotypes differ in speed of emergence and root architecture. While AgP4 germinates faster and shows a tap root-dominant phenotype with a high proportion of root hairs, AgP1 is slower in germination and has a more pronounced lateral root system. Both genotypes were "00" hybrids. B. napus seeds were surface-sterilized with 3\% sodium hypochlorite solution (Carl Roth) for $15 \mathrm{~min}$ and were thoroughly washed three times with sterile deionized water. Surface-sterilized seeds were plated on sterile glass Petri dishes fitted with filter papers (grade MN 619 eh) (Macherey-Nagel), which were soaked with autoclaved water. The seed plates were incubated for $12 \mathrm{~h}$ at $8^{\circ} \mathrm{C}$ in the dark. Subsequently, the plates were transferred into a growth chamber with $16 \mathrm{~h}$ of light $\left(80 \mu \mathrm{mol} \mathrm{m} \mathrm{m}^{-2} \mathrm{~s}^{-1}\right)$ and $8 \mathrm{~h}$ of dark, at $20^{\circ} \mathrm{C}$ and $60 \%$ relative humidity for germination. Six-dayold single seedlings were transferred into sterile boxes fitted with sterile filter papers soaked with half-strength MS medium (Sigma-Aldrich) supplemented with $0.5 \%$ sucrose and $5.4 \mu \mathrm{M}$ NAA (1-naphthylacetic acid). These plant boxes were placed in an incubator set to the conditions mentioned above. Roots of 14-day-old seedlings were directly treated on filter paper with deionized water (control), OMG16, FZB42, or OMG16 plus FZB42. Prior to inoculation, an approximately 2-cm arch was formed with the roots to prevent spreading of the microorganisms on the filter paper (Supplementary Fig. S5). For single fungal inoculation, plants were treated with $10-\mu$ l fungal spore suspensions (200 spores per root) dropped directly onto each arch of roots (drop inoculation). For single bacterial inoculation, roots were inoculated with $10 \mu \mathrm{l}$ of FZB42 culture with an $\mathrm{OD}_{600}$ of 0.23 (corresponding to approximately $1.8 \times 10^{8}$ cells per milliliter), as estimated by using Agilent's Escherichia coli cell culture concentration from the $\mathrm{OD}_{600}$ calculator. For coinoculation, single roots were inoculated with a $20-\mu$ l mixture of OMG16 and FZB42 solution. Five biological replicates were collected for each treatment.

\section{Microscopy.}

To analyze rapeseed root colonization by $T$. harzianum., 14-day-old rapeseed seedlings were inoculated with OMG16 and OMG16 plus FZB42. Prior to staining, roots were separated from the stem with a sharp scalpel and were thoroughly washed with tap water. Then, the roots were heated in $10 \%$ $\mathrm{KOH}$ for $3 \mathrm{~min}$ at $121^{\circ} \mathrm{C}$. After heating, roots were washed repeatedly in tap water. Afterward, roots were soaked in $1 \%$ $\mathrm{HCl}$ for $1 \mathrm{~h}$ and were then stained with fuchsine red solution $(0.01 \%$ acidic fuchsin, $87.5 \%$ lactic acid, and $6.3 \%$ glycerol in $\mathrm{H}_{2} \mathrm{O}$ ) for $3 \mathrm{~min}$ at $121^{\circ} \mathrm{C}$. Lastly, the roots were stored in $50 \%$ glycerol and were visualized under a Zeiss Axio Observer 7 inverse microscope, using the ZEN 2.0 imaging software (Carl Zeiss).

\section{Primer design.}

Initially, the primer sequences of Bacillus velezensis FZB42 strain-specific marker and two ISR genes, AOC3 and ERF2, were taken from Borriss (2011) and Alkooranee et al. (2017), respectively. The targets were amplified and sequenced (Microsynth Seqlab), and internal primer sequences were designed, using the Primer 3 software (Rozen and Skaletsky 2000). In order to design VSP2 and BnaUbiquitin11 primer pairs, a total of five sequences of BnaUbiquitin11 gene transcripts and two sequences of VSP2 transcripts were taken from the National Center for Biotechnology Information database. Consensus sequences were generated from five and two entries, respectively, using BioEdit Sequence Editor, version 7.0.5 (Ibis Biosciences), and were used to design primer pairs. OMG16 primers were designed from a distinct microsatellite locus $(\mathrm{AGT})_{13}$. Its sequence is shown in Supplementary Figure S6. All primer sequences used in this study are listed in Supplementary Table S7.

\section{Quantification of OMG16 root colonization.}

The relative amount of root endophytic OMG16 fungal DNA in the presence or absence of FZB42 bacterium inside rapeseed roots was determined in total root DNA by qPCR, using the absolute quantification approach, based on a standard curve. Total DNA was extracted from roots of plants inoculated with OMG16 and OMG16 plus FZB42. Approximately 3-cm root sections were cut from drop-inoculated samples with a sharp scalpel, were washed with sterile deionized water by vortexing for $30 \mathrm{~s}$, were rinsed with water, were dried between soft tissue papers, and were cut into small pieces. Total DNA was isolated from approximately $50 \mathrm{mg}$ of root segments with the DNeasy plant mini kit (Qiagen) according to the manufacturer instructions. DNA quality was assayed on $0.9 \%$ agarose gels and concentrations were determined with the Qubit 3.0 fluorometer using the Qubit dsDNA assay kit (Thermo Fisher Scientific). 
qPCR was carried out in $20-\mu \mathrm{l}$ reaction volumes containing $15 \mathrm{ng}$ of extracted root DNA, $10 \mu \mathrm{l}$ of PowerUp SYBR Green master mix (Applied Biosystems), the forward and reverse primers, at final concentrations of 5 pmol each, and nuclease-free PCR-grade water to adjust the final volume. Strain-specific markers for OMG16 (T.harz.SSRAGT13-F/R) and FZB42 (FZB42-F/R) were applied for absolute quantification of the resulting amplicons. Amplifications were performed in a QuantStudio 5 real-time PCR system (Applied Biosystems) under the following conditions: $50^{\circ} \mathrm{C}$ for $2 \mathrm{~min}, 95^{\circ} \mathrm{C}$ for $2 \mathrm{~min}$, and 40 cycles of amplification (denaturation, $95^{\circ} \mathrm{C}$ for $1 \mathrm{~s}$; annealing and elongation, $60^{\circ} \mathrm{C}$ for $\mathrm{OMG} 16$ or $63^{\circ} \mathrm{C}$ for $\mathrm{FZB} 42$, for $20 \mathrm{~s}$ ). A melting curve was recorded with initial denaturation at $95^{\circ} \mathrm{C}$ for $1 \mathrm{~s}$, annealing at $60^{\circ} \mathrm{C}$ for $20 \mathrm{~s}$, and finally, a linear increase of the temperature from 60 to $95^{\circ} \mathrm{C}$ with a rate of $0.1^{\circ} \mathrm{C} \mathrm{s}^{-1}$. qPCR was performed in technical quadruplicate for each biological replicate. Standard curves were determined with dilution series of pure OMG16 and FZB42 DNAs in the range of $10 \mathrm{ng}$ to $1 \mathrm{pg}$. The absolute amount of OMG16 and FZB42 DNA in total root DNA was calculated from quantification cycles $(\mathrm{Cq})$, using the standard curves. Copy numbers of OMG16 strainspecific genomic region were calculated from the absolute amount of OMG16 DNA. There were no cross reactions with any of the bacterial or plant DNAs used.

\section{Observation of root morphology.}

Roots of 2-week-old rapeseed seedlings were inoculated with OMG16, FZB42, and OMG16 plus FZB42 strains by dropinoculation or were treated with distilled water for control according to the procedure described above. Five biological replicates were assigned for each treatment and cultivar, and care was taken that seedlings of identical developmental stage and size were used for the experiment. Roots were harvested on three consecutive days after inoculation and were stored in $30 \%$ (vol/vol) ethanol until further use. The root systems were spread in a film of water in transparent Perspex trays and images were taken with a flat-bed scanner (Epson Expression 10000 XL). Root morphological characteristics were measured with the WinRHIZO Pro V.2009c image analysis system (Reagent Instruments, WinRHIZO software release 2009). Afterward, the roots were oven dried at $65^{\circ} \mathrm{C}$ for $48 \mathrm{~h}$, in order to assess the dry weights.

\section{Gene-expression studies upon beneficial microbial treatments.}

The green plant parts from root colonization experiments were immediately transferred to $-80^{\circ} \mathrm{C}$ at 1 hai, and 1,2 , and 3 dai. Total RNA was isolated from approximately $60 \mathrm{mg}$ of leaves, using the RNeasy plant mini kit (Qiagen), according to the manufacturer instructions with the following modifications. Plant material was ground in a speed mill (FastPrep 24, MP Biomedicals) with $450 \mu \mathrm{l}$ of Qiagen lysis buffer containing $1 \%$ 2-mercaptoethanol. Approximately $350 \mu \mathrm{l}$ of clear lysates were obtained and were aliquoted into $50 \mu \mathrm{l}$ each for DNase treatment. Turbo DNase $(1 \mu \mathrm{l})$ (TURBO DNA-free kit, Thermo Fisher Scientific) was added twice in each $50-\mu 1$ lysate aliquot and was incubated at $37^{\circ} \mathrm{C}$ for 20 and $10 \mathrm{~min}$, respectively. DNase inactivation reagent $(20 \mu \mathrm{l}$ per tube) was added to stop the enzymatic reaction and to remove divalent cations. RNA extraction was continued according to the instructions of the RNeasy plant mini kit (Qiagen). RNA concentrations were quantified with a Qubit 3.0 fluorometer using the Qubit RNA HS assay kit (Thermo Fisher Scientific). cDNA was synthesized from $1.2 \mu \mathrm{g}$ of total RNA, using the SuperScript IV first-strand synthesis system (Thermo Fisher Scientific). cDNA concentrations were measured with a Qubit 3.0 fluorometer, using the Qubit ssDNA assay kit (Thermo Fisher Scientific).
Gene expression was analyzed by qRT-PCR based on relative transcript abundances. The ideal annealing temperature (Supplementary Table S7) of each primer pair was determined by gradient PCR and was adopted in qRT-PCR, which was performed in a QuantStudio 5 real-time PCR system (Applied Biosystems) with PowerUp SYBR Green master mix (Applied Biosystems). All PCR reactions contained $65 \mathrm{ng}$ of cDNA, $10 \mu \mathrm{l}$ of master mix, and 5 pmol each of forward and reverse primers and were carried out in quadruplicate in $20 \mu \mathrm{l}$ of total volume under the following conditions: $50^{\circ} \mathrm{C}$ for $2 \mathrm{~min}, 95^{\circ} \mathrm{C}$ for $2 \mathrm{~min}$, and 40 cycles of amplification (denaturation, $95^{\circ} \mathrm{C}$ for $1 \mathrm{~s}$; annealing and elongation, $60^{\circ} \mathrm{C}$ for $\left.30 \mathrm{~s}\right)$. After amplification, a melting curve was recorded as described above. Standard curves with the amplification efficiency of each primer pair and melting curves of generated PCR products are shown in Supplementary Figure S8. BnaUbiquitin11, BnaActin, and BnaTubulin were used as endogenous controls for normalization. Relative transcript abundances were calculated using the Pfaffl and Vandesompele methods (Pfaffl 2001; Vandesompele et al. 2002) and MIQE guidelines for qRT-PCR (Bustin et al. 2009). Briefly, efficiency corrected $\mathrm{Cq}$ of three endogenous controls were averaged and $\Delta \mathrm{Cq}(\mathrm{Cq}$ reference genes minus $\mathrm{Cq}$ target gene) was calculated.

\section{Infection of rapeseed roots with $\mathrm{Vl43.}$}

B. napus seeds of the AgP4 cultivar were grown following the procedure described above. Two-week-old rapeseed roots were taken from the sterile boxes and were incubated by dipping for $30 \mathrm{~min}$ in a mixed suspension of OMG16 (2,000 spores per milliliter) plus FZB42 $\left(\mathrm{OD}_{600}=0.275\right.$, corresponding to approximately $2.2 \times 10^{8}$ cells per milliliter) or in deionized water (control). Subsequently, plants were placed back in the boxes and were incubated in a growth chamber set to the conditions mentioned above. Two days after OMG16 plus FZB42 treatment, roots of the primed plants were infected by dipping them into VI43 spore suspension $\left(10^{6}\right.$ spores per milliliter) for $30 \mathrm{~min}$ or into deionized water (control). As positive controls, roots of nonprimed AgP4 seedlings were infected by dipping in V143 spores for $30 \mathrm{~min}$. For each treatment, three individual plants were pooled to obtain one biological replicate and five biological replicates were collected for each treatment.

\section{Quantification of V143 DNA.}

The absolute amount of V143 DNA present in total root DNA of V143- and OMG16 plus FZB42 plus V143-treated plants were quantified. qPCR analysis was performed by following the protocol mentioned above with $15 \mathrm{ng}$ of DNA isolated from infected roots and the $V$. longisporum-specific primer pairs OLG70 and OLG71 (Eynck et al. 2007).

\section{Relative expression of defense-related genes after V143 infection.}

Relative expression of rapeseed defense genes upon V143 pathogen challenge was analyzed by qRT-PCR. Total RNA was isolated from leaves. qRT-PCR analysis was performed with $15 \mathrm{ng}$ of cDNA according to the protocol described above.

\section{Statistical analysis.}

All calculations were carried out with $\mathrm{R}$ software, version 4.0.2 (R Development Core Team 2019). In the root colonization experiment with OMG16, a Shapiro-Wilk normality test was performed. All data sets were normally distributed except the copy number of OMG16 at 1 dai in AgP4 cultivar. Thus, a Wilcoxon rank-sum test was employed at $P<0.05$ at 1 dai for the AgP4 cultivar and the rest of the data were analyzed with Welch's $t$ test at $P<0.05$. Observation of root morphology was analyzed by Dunnett's test with multivariate testing (mvt) 
adjustment at $P<0.05$. Significant differences in transcript abundances were also calculated by Dunnett's test with mvt adjustment at $P<0.05$. The differences in gene expression upon single and combined microbial treatments were analyzed by one-way analysis of variance with post hoc Tukey's highly significant difference tests. Unpaired $t$ test was performed at $P<0.05$, to analyze the amount of V143 in rapeseed roots. Significant differences were denoted using asterisks as follows: one asterisk to indicate $P<0.05$, two $P<0.01$, three $P<$ 0.001 , and four $P<0.0001$.

\section{ACKNOWLEDGMENTS}

The authors would like to thank M. Lakishyk and S. Grabner of IBAS, Bernburg for excellent technical support.

\section{AUTHOR-RECOMMENDED INTERNET RESOURCES}

The $\mathrm{OD}_{600}$ calculator:

https://www.chem.agilent.com/store/biocalculators/calcODBacterial.jsp

National Center for Biotechnology Information database:

https://www.ncbi.nlm.nih.gov

\section{LITERATURE CITED}

Alizadeh, H., Behboudi, K., Ahmadzadeh, M., Javan-Nikkhah, M., Zamioudis, C., Pieterse, C. M. J., and Bakker, P. A. H. M. 2013. Induced systemic resistance in cucumber and Arabidopsis thaliana by the combination of Trichoderma harzianum Tr6 and Pseudomonas sp. Ps14. Biol. Control 65:14-23.

Alkooranee, J. T., Aledan, T. R., Ali, A. K., Lu, G., Zhang, X., Wu, J., $\mathrm{Fu}, \mathrm{C}$., and Li, M. 2017. Detecting the hormonal pathways in oilseed rape behind induced systemic resistance by Trichoderma harzianum TH12 to Sclerotinia sclerotiorum. PLoS One 12:e0168850.

Alkooranee, J. T., Aledan, T. R., Xiang, J., Lu, G., and Li, M. 2015. Induced systemic resistance in two genotypes of Brassica napus (AACC) and Raphanus oleracea (RRCC) by Trichoderma isolates against Sclerotinia sclerotiorum. Am. J. Plant Sci. 6:1662-1674.

Alonso-Ramírez, A., Poveda, J., Martín, I., Hermosa, R., Monte, E., and Nicolás, C. 2015. Trichoderma harzianum root colonization in Arabidopsis. Bio Protoc. 5:e1512.

Angel, L. P. L., Yusof, M. T., Ismail, I. S., Ping, B. T. Y., Mohamed Azni, I. N., Kamarudin, N. H. J., and Sundram, S. 2016. An in vitro study of the antifungal activity of Trichoderma virens $7 \mathrm{~b}$ and a profile of its non-polar antifungal components released against Ganoderma boninense. J. Microbiol. 54:732-744.

Berg, G., Rybakova, D., Grube, M., and Köberl, M. 2016. The plant microbiome explored: Implications for experimental botany. J. Exp. Bot. 67:995-1002.

Borriss, R. 2011. Use of plant-associated Bacillus strains as biofertilizers and biocontrol agents in agriculture. Pages 41-76 in: Bacteria in Agrobiology: Plant Growth Responses. D. K. Maheshwari, ed. Springer Verlag, Berlin.

Brotman, Y., Landau, U., Cuadros-Inostroza, Á., Tohge, T., Fernie, A. R., Chet, I., Viterbo, A., and Willmitzer, L. 2013. Trichodermaplant root colonization: Escaping early plant defense responses and activation of the antioxidant machinery for saline stress tolerance. PLoS Pathog. 9:e1003221.

Bustin, S. A., Benes, V., Garson, J. A., Hellemans, J., Huggett, J., Kubista, M., Mueller, R., Nolan, T., Pfaffl, M. W., Shipley, G. L., Vandesompele, J., and Wittwer, C. T. 2009. The MIQE guidelines: Minimum information for publication of quantitative real-time PCR experiments. Clin. Chem. 55:611-622.

Carro-Huerga, G., Compant, S., Gorfer, M., Cardoza, R. E., Schmoll, M., Gutiérrez, S., and Casquero, P. A. 2020. Colonization of Vitis vinifera L. by the endophyte Trichoderma sp. Strain T154: Biocontrol activity against Phaeoacremonium minimum. Front. Plant Sci. 11:1170.

Chacón, M. R., Rodríguez-Galán, O., Benítez, T., Sousa, S., Rey, M., Llobell, A., and Delgado-Jarana, J. 2007. Microscopic and transcriptome analyses of early colonization of tomato roots by Trichoderma harzianum. Int. Microbiol. 10:19-27.

Chanchaichaovivat, A., Panijpana, B., and Ruenwongsaab, P. 2008. Putative modes of action of Pichia guilliermondii strain R13 in controlling chilli anthracnose after harvest. Biol. Control 47:207-215.
Chowdhury, S. P., Hartmann, A., Gao, X., and Borriss, R. 2015. Biocontrol mechanism by root-associated Bacillus amyloliquefaciens FZB42-A review. Front. Microbiol. 6:780.

Coleman-Derr, D., Desgarennes, D., Fonseca-Garcia, C., Gross, S., Clingenpeel, S., Woyke, T., North, G., Visel, A., Partida-Martinez, L. P., and Tringe, S. G. 2016. Plant compartment and biogeography affect microbiome composition in cultivated and native Agave species. New Phytol. 209:798-811.

Coppola, M., Cascone, P., Lelio, I. D., Woo, S. L., Lorito, M., Rao, R., Pennacchio, F., Guerrieri, E., and Digilio, M. C. 2019. Trichoderma atroviride $\mathrm{P} 1$ colonization of tomato plants enhances both direct and indirect defense barriers against insects. Front. Physiol. 10:813.

Depotter, J. R. L., Deketelaere, S., Inderbitzin, P., Tiedemann, A. V. Höfte, M., Subbarao, K. V., Wood, T. A., and Thomma, B. P. H. J. 2016. Verticillium longisporum, the invisible threat to oilseed rape and other brassicaceous plant hosts. Mol. Plant Pathol. 17:1004-1016.

Doornbos, R. F., van Loon, L. C., and Bakker, P. A. 2012. Impact of root exudates and plant defense signaling on bacterial communities in the rhizosphere. A review. Agron. Sustain. Dev. 32:227-243.

Eltayeb Habora, M. E., Eltayeb, A. E., Oka, M., Tsujimoto, H., and Tanaka, K. 2013. Cloning of allene oxide cyclase gene from Leymus mollis and analysis of its expression in wheat-Leymus chromosome addition lines. Breed. Sci. 63:68-76.

Eynck, C., Koopmann, B., Grunewaldt-Stoecker, G., Karlovsky, P., and Tiedemann, A. 2007. Differential interactions of Verticillium longisporum and $V$. dahliae with Brassica napus detected with molecular and histological techniques. Eur. J. Plant Pathol. 118:259-274.

Eynck, C., Koopmann, B., Karlovsky, P., and von Tiedemann, A. 2009. Internal resistance in winter oilseed rape inhibits systemic spread of the vascular pathogen Verticillium longisporum. Phytopathology 99 802-811.

Fan, B., Borriss, R., Bleiss, W., and Wu, X. 2012. Gram-positive rhizobacterium Bacillus amyloliquefaciens FZB42 colonizes three types of plants in different patterns. J. Microbiol. 50:38-44.

Fan, B., Chen, X. H., Budiharjo, A., Bleiss, W., Vater, J., and Borriss, R. 2011. Efficient colonization of plant roots by the plant growth promoting bacterium Bacillus amyloliquefaciens FZB42, engineered to express green fluorescent protein. J. Biotechnol. 151:303-311.

Fan, B., Wang, C., Song, X., Ding, X., Wu, L., Wu, H., Gao, X., and Borriss, R. 2018. Bacillus velezensis FZB42 in 2018: The grampositive model strain for plant growth promotion and biocontrol. Front. Microbiol. 9:2491.

Frey-Klett, P., Garbaye, J., and Tarkka, M. 2007. The mycorrhiza helper bacteria revisited. New Phytol. 176:22-36.

Geistlinger, J., Zwanzig, J., Heckendorff, S., and Schellenberg, I. 2015. SSR markers for Trichoderma virens: Their evaluation and application to identify and quantify root-endophytic strains. Diversity (Basel) 7: 360-384.

Han, Q., Wu, F., Wang, X., Qi, H., Shi, L., Ren, A., Liu, Q., Zhao, M., and Tang, C. 2015. The bacterial lipopeptide iturins induce Verticillium dahliae cell death by affecting fungal signalling pathways and mediate plant defence responses involved in pathogen-associated molecular pattern-triggered immunity. Environ. Microbiol. 17:1166-1188.

Harman, G. E., Howell, C. R., Viterbo, A., Chet, I., and Lorito, M. 2004. Trichoderma species-Opportunistic, avirulent plant symbionts. Nat Rev. Microbiol. 2:43-56.

Heil, M., and Bostock, R. M. 2002. Induced systemic resistance (ISR) against pathogens in the context of induced plant defences. Ann. Bot. 89:503-512.

Hermosa, R., Viterbo, A., Chet, I., and Monte, E. 2012. Plant-beneficial effects of Trichoderma and of its genes. Microbiology (Reading) 158: 17-25.

Hoyos-Carvajal, L., Orduz, S., and Bisset, J. 2009. Growth stimulation in bean (Phaseolus vulgaris L.) by Trichoderma. Biol. Control 51:409-416.

Jacquard, C., Mazeyrat-Gourbeyre, F., Devaux, P., Boutilier, K., Baillieul, F., and Clément, C. 2009. Microspore embryogenesis in barley: Anther pre-treatment stimulates plant defence gene expression. Planta 229:393-402.

Karapapa, V. K., Bainbridge, B. W., and Heale, J. B. 1997. Morphological and molecular characterization of Verticillium longisporum comb. nov., pathogenic to oilseed rape. Mycol. Res. 101:1281-1294.

Karlsson, M., Atanasova, L., Jensen, D. F., and Zeilinger, S. 2017. Necrotrophic mycoparasites and their genomes. Microbiol. Spectr. 5:10.

Karuppiah, V., Sun, J., Li, T., Vallikkannu, M., and Chen, J. 2019. Cocultivation of Trichoderma asperellum GDFS1009 and Bacillus amyloliquefaciens 1841 causes differential gene expression and improvement in the wheat growth and biocontrol activity. Front. Microbiol. 10:1068. 
Karuppiah, V., Zhixiang, L., Liu, H., Vallikkannu, M., and Chen, J. 2021. Co-culture of Vell-overexpressed Trichoderma asperellum and Bacillus amyloliquefaciens: An eco-friendly strategy to hydrolyze the lignocellulose biomass in soil to enrich the soil fertility, plant growth and disease resistance. Microb. Cell Fact. 20:57.

Khoshmanzar, E., Aliasgharzad, N., Neyshabouri, M. R., Khoshru, B., Arzanlou, M., and Lajayeral, B. A. 2020. Effects of Trichoderma isolates on tomato growth and inducing its tolerance to water-deficit stress. Int. J. Environ. Sci. Technol. 17:869-878.

Kloepper, J. W., Leong, J., Teintze, M., and Schroth, M. N. 1980 Enhancing plant growth by siderophores produces by plant-growthpromoting rhizobacteria. Nature 286:885-886.

Köhl, J., Kolnaar, R., and Ravensberg, W. J. 2019. Mode of action of microbial biological control agents against plant diseases: Relevance beyond efficacy. Front. Plant Sci. 10:845.

Koscielny, C. B., and Gulden, R. H. 2012. Seedling root length in Brassica napus L. is indicative of seed yield. Can. J. Plant Pathol. 92: 1229-1237.

Lee, B., Farag, M. A., Park, H. B., Kloepper, J. W., Lee, S. H., and Ryu, C.-M. 2012. Induced resistance by a long-chain bacterial volatile: Elicitation of plant systemic defense by a C13 volatile produced by Paenibacillus polymyxa. PLoS One 7:e48744.

Liu, B., Wang, W., Gao, J., Chen, F., Wang, S., Xu, Y., Tang, L., and Jia, Y. 2010. Molecular cloning and characterization of a jasmonate biosynthetic pathway gene for allene oxide cyclase from Jatropha curcas. Acta Physiol. Plant. 32:531-539.

Louvieaux, J., Spanoghe, M., and Hermans, C. 2020. Root morphological traits of seedlings are predictors of seed yield and quality in winter oilseed rape hybrid cultivars. Front. Plant Sci. 11:568009.

Mendes, R., Kruijt, M., de Bruijn, I., Dekkers, E., van der Voort, M., Schneider, J. H., Piceno, Y. M., DeSantis, T. Z., Andersen, G. L., Bakker, P. A., and Raaijmakers, J. M. 2011. Deciphering the rhizosphere microbiome for disease-suppressive bacteria. Science 332: 1097-1100.

Meng, X., Miao, Y., Liu, Q., Ma, L., Guo, K., Liu, D., Ran, W., and Shen, Q. 2019. TgSWO from Trichoderma guizhouense NJAU4742 promotes growth in cucumber plants by modifying the root morphology and the cell wall architecture. Microb. Cell Fact. 18:148.

Moradtalab, N., Ahmed, A., Geistlinger, J., Walker, F., Höglinger, B. Ludewig, U., and Neumann, G. 2020. Synergisms of microbial consortia, $\mathrm{N}$ forms, and micronutrients alleviate oxidative damage and stimulate hormonal cold stress adaptations in maize. Front. Plant Sci. 11: 396.

Mpanga, I. K., Gomez-Genao, N., Moradtalab, N., Wanke, D. Chrobaczek, V., Ahmed, A., Windisch, S., Geistlinger, J., Hafiz, F. B., Walker, F., Ludewig, U., and Neumann, G. 2019b. The role of $\mathrm{N}$ form supply for PGPM-host plant interactions in maize. J. Plant Nutr. Soil Sci. 182:908-920

Mpanga, I. K., Ludewig, U., Dapaah, H. K., and Neumann, G. 2020 Acquisition of rock phosphate by combined application of ammonium fertilizers and Bacillus amyloliquefaciens FZB42 in maize as affected by soil pH. J. Appl. Microbiol. 129:947-957.

Mpanga, I. K., Nkebiwe, P. M., Kuhlmann, M., Cozzolino, V., Piccolo, A., Geistlinger, J., Berger, N., Ludewig, U., and Neumann, G. 2019a. The form of $\mathrm{N}$ supply determines plant growth promotion by P-solubilizing microorganisms in maize. Microorganisms 7:38.

Naznin, H. A., Kiyohara, D., Kimura, M., Miyazawa, M., Shimizu, M., and Hyakumachi, M. 2014. Systemic resistance induced by volatile organic compounds emitted by plant growth-promoting fungi in Arabidopsis thaliana. PLoS One 9:e86882.

Palyzová, A., Svobodová, K., Sokolová, L., Novák, J., and Novotný, Č. 2019. Metabolic profiling of Fusarium oxysporum f. sp. conglutinans race 2 in dual cultures with biocontrol agents Bacillus amyloliquefaciens, Pseudomonas aeruginosa, and Trichoderma harzianum. Folia Microbiol. (Praha) 64:779-787.

Pelagio-Flores, R., Esparza-Reynoso, S., Garnica-Vergara, A., LópezBucio, J., and Herrera-Estrella, A. 2017. Trichoderma-induced acidification is an early trigger for changes in Arabidopsis root growth and determines fungal phytostimulation. Front. Plant Sci. 8:822.

Pfaffl, M. W. 2001. A new mathematical model for relative quantification in real-time RT-PCR. Nucleic Acids Res. 29:e45.

Poveda, J., Hermosa, R., Monte, E., and Nicolás, C. 2019. Trichoderma harzianum favours the access of arbuscular mycorrhizal fungi to nonhost Brassicaceae roots and increases plant productivity. Sci. Rep. 9: 11650.

R Development Core Team. 2019. A language and environment for statistical computing. R Foundation for Statistical Computing, Vienna.
Rodriguez, R. J., White, J. F., Jr., Arnold, A. E., and Redman, R. S. 2009. Fungal endophytes: Diversity and functional roles. New Phytol. 182:314-330.

Rozen, S., and Skaletsky, H. 2000. Primer3 on the WWW for general users and for biologist programmers. Methods Mol. Biol. 132:365 386.

Rybakova, D., Mancinelli, R., Wikström, M., Birch-Jensen, A.-S., Postma, J., Ehlers, R.-U., Goertz, S., and Berg, G. 2017. The structure of the Brassica napus seed microbiome is cultivar-dependent and affects the interactions of symbionts and pathogens. Microbiome 5:104.

Samolski, I., Rincón, A. M., Pinzón, L. M., Viterbo, A., and Monte, E. 2012. The qid74 gene from Trichoderma harzianum has a role in root architecture and plant biofertilization. Microbiology (Reading) 158: 129-138.

Sarosh, B. R., Danielsson, J., and Meijer, J. 2009. Transcript profiling of oilseed rape (Brassica napus) primed for biocontrol differentiate genes involved in microbial interactions with beneficial Bacillus amyloliquefaciens from pathogenic Botrytis cinerea. Plant Mol. Biol. 70:31-45.

Schaller, F., Zerbe, P., Reinbothe, S., Reinbothe, C., Hofmann, E., and Pollmann, S. 2008. The allene oxide cyclase family of Arabidopsis thaliana: Localization and cyclization. FEBS J. 275:2428-2441.

Segarra, G., Casanova, E., Avilés, M., and Trillas, I. 2010. Trichoderma asperellum strain T34 controls fusarium wilt disease in tomato plants in soilless culture through competition for iron. Microb. Ecol. 59:141-149.

Stenzel, I., Otto, M., Delker, C., Kirmse, N., Schmidt, D., Miersch, O., Hause, B., and Wasternack, C. 2012. ALLENE OXIDE CYCLASE (AOC) gene family members of Arabidopsis thaliana: Tissue- and organ-specific promoter activities and in vivo heteromerization. J. Exp. Bot. 63:6125-6138

Thonar, C., Lekfeldt, J. D. S., Cozzolino, V., Kundel, D., Kulhánek, M., Mosimann, C., Neumann, G., Piccolo, A., Rex, M., Symanczik, S., Walder, F., Weinmann, M., de Neergaard, A., and Mäder, P. 2017. Potential of three microbial bio-effectors to promote maize growth and nutrient acquisition from alternative phosphorous fertilizers in contrasting soils. Chem. Biol. Technol. Agric. 4:7.

Torres, M. J., Brandan, C. P., Petroselli, G., Erra-Balsells, R., and Audisio, M. C. 2016. Antagonistic effects of Bacillus subtilis subsp. subtilis and B. amyloliquefaciens against Macrophomina phaseolina: SEM study of fungal changes and UV-MALDI-TOF MS analysis of their bioactive compounds. Microbiol. Res. 182:31-39.

Tucci, M., Ruocco, M., De Masi, L., De Palma, M., and Lorito, M. 2011. The beneficial effect of Trichoderma spp. on tomato is modulated by the plant genotype. Mol. Plant Pathol. 12:341-354.

Vandesompele, J., De Preter, K., Pattyn, F., Poppe, B., Van Roy, N., De Paepe, A., and Speleman, F. 2002. Accurate normalization of real-time quantitative RT-PCR data by geometric averaging of multiple internal control genes. Genome Biol. 3:research0034.1.

Velázquez-Robledo, R., Contreras-Cornejo, H. A., Macías-Rodríguez, L. Hernández-Morales, A., Aguirre, J., Casas-Flores, S., López-Bucio, J., and Herrera-Estrella, A. 2011. Role of the 4-phosphopantetheinyl transferase of Trichoderma virens in secondary metabolism and induction of plant defense responses. Mol. Plant-Microbe Interact 24:1459-1471.

Viterbo, A., and Chet, I. 2006. TasHyd1, a new hydrophobin gene from the biocontrol agent Trichoderma asperellum, is involved in plant root colonization. Mol. Plant Pathol. 7:249-258.

White, J. F., Kingsley, K. L., Verma, S. K., and Kowalski, K. P. 2018. Rhizophagy cycle: An oxidative process in plants for nutrient extraction from symbiotic microbes. Microorganisms 6:95.

Wilhelm, S. 1955. Longevity of the Verticillium wilt fungus in the laboratory and field. Phytopathology 45:180-181.

Windisch, S., Sommermann, L., Babin, D., Chowdhury, S. P., Grosch, R., Moradtalab, N., Walker, F., Höglinger, B., El-Hasan, A., Armbruster, W., Nesme, J., Sørensen, S. J., Schellenberg, I., Geistlinger, J., Smalla, K., Rothballer, M., Ludewig, U., and Neumann, G. 2021. Impact of long-term organic and mineral fertilization on rhizosphere metabolites, root-microbial interactions and plant health of lettuce. Front. Microbiol. 11:597745.

Wu, L., Huang, Z., Li, X., Ma, L., Gu, Q., Wu, H., Liu, J., Borriss, R., Wu, Z., and Gao, X. 2018. Stomatal closure and SA-, JA/ET- signaling pathways are essential for Bacillus amyloliquefaciens FZB42 to restrict leaf disease caused by Phytophthora nicotianae in Nicotiana benthamiana. Front. Microbiol. 9:847.

Wu, Q., Wu, J., Sun, H., Zhang, D., and Yu, D. 2011. Sequence and expression divergence of the AOC gene family in soybean: Insights into functional diversity for stress responses. Biotechnol. Lett. 33: 1351-1359. 
Yang, Y., Dong, C., Li, X., Du, J., Qian, M., Sun, X., and Yang, Y. 2016. A novel Ap2/ERF transcription factor from Stipa purpurea leads to enhanced drought tolerance in Arabidopsis thaliana. Plant Cell Rep. 35:2227-2239.

Yedidia, I., Benhamou, N., and Chet, I. 1999. Induction of defense responses in cucumber plants (Cucumis sativus L.) By the biocontrol agent trichoderma harzianum. Appl. Environ. Microbiol. 65:1061-1070.

Yedidia, I., Benhamou, N., Kapulnik, Y., and Chet, I. 2000. Induction and accumulation of PR proteins activity during early stages of root colonization by the mycoparasite Trichoderma harzianum strain T203. Plant Physiol. Biochem. 38:863-873.

Yusran, Y., Roemheld, V., and Mueller, T. 2009. Effects of Pseudomonas sp. "Proradix" and Bacillus amyloliquefaciens FZB42 on the establishment of AMF infection, nutrient acquisition and growth of tomato affected by Fusarium oxysporum Schlecht f.sp. radicislycopersici Jarvis and Shoemaker. The Proceedings of the International Plant Nutrition Colloquium XVI, UC Davis: Department of Plant Sciences, Davis, CA, U.S.A.

Zhang, D., Spadaro, D., Valente, S., Garibaldi, A., and Gullino, M. L. 2011. Cloning, characterization and expression of an exo-1,3- $\beta$-glucanase gene from the antagonistic yeast, Pichia guilliermondii strain M8 against grey mold on apples. Biol. Control 59:284-293.

Zhao, Y., Dong, W., Zhang, N., Ai, X., Wang, M., Huang, Z., Xiao, L., and Xia, G. 2014. A wheat allene oxide cyclase gene enhances salinity tolerance via jasmonate signaling. Plant Physiol. 164:1068-1076. 